\section{La Educación de Enfermería en el Estado Capitalista Ecuatoriano: 1960-1983.}

Patricia de la Torre A.* Margarita Velasco Abad**
En este articulo, las autoras descibren la transformación de la enseñanza y práctica de la enfermeria en el Ecuador, desde su etapa inicial, con la introducción de éste por de personal extranjero através de agencias internacionales, hasta la substitución de éste por personal ecuatoriano profesionalizado. Simultáneamente, se estudia el proceso de integración de las escuelas de enfermeria en la estructura universitaria, y se analizan, también, aciertos y desaciertos del Estado, la Universidad y los organismos internacionales de salud en la elaboración de una politica sanitaria unificada que atienda las necesidades de la población, $y$ del papel que, em todo ello, le cabria desempeñar a la enfermeria.

\section{1}

La transición del Estado Liberal al moderno Estado capita. lista de hoy, inicia una nueva etapa en 1963 cuando se ponen las bases para el desarrollismo. Para poder caracterizar esta transición y el impacto que esta opera en las políticas de salud y de educación de enfermeria, hemos considerado tres períodos:

Primer Período: 1960-1970: El Estado desarrollista, la adopción de una política de salud y la transición de la educación de enfermería a carrera universitaria.

Segundo Período: 1970-1977: La Modernización del Estado, la politica de Extensión de Cobertura y la consolidación de la professionalización de al enfermera a través de la Primeira Politica educativa emitida por la Asociación Ecuatoriana de Escuelas de Enfermería (ASEDEE).

Tercer Período: 1977.1983: El Estado Democrático; la tendencia comunitaria en salud y su influencia en la adopción de la Segunda Política de Educación de Enfermería.

Algunos de los datos que aparecen en al presente artículo son extraidos del trabajo de investigación titulado: "La Educación de Enfermería en el Ecuador: Notas para su Cademos de Saúde Pública, R.J., 2(4): 167-196, abr/jun, 1988.
* Professora de la Facultad de Enfermería de la Pontificia Universidad Católica del Ecua. dor y Coordinadora del Centro de Investigación y Tecnología (CITE) de la Facultad.

** Projessora de la Escuela Nacio. nal de Enfermería de la Facul. tad de Ciencias Médicas de la Universidad Central del Ecuador. 
análisis" realizado por las autoras, bajo el auspicio de ASEDEE, en mayo de 1983.

\section{PRIMER PERIODO: 1960-1970}

Para esta década el Ecuador se encuentra en una profunda crisis económica debido a la baja de exportaciones del banano. La salida adoptada es reforzar y viabilizar un proceso de industrialización, cuyos interese están claramente diferenciados; así los exportadores tratan de encontrar un sector productivo al que puedan transferir sus inversiones y por lo tanto apoyar el modelo desarrollista, cuyo principal organismo técnico es la Junta Nacional de Planificación (Velasco, Fernando; 1981:205).

Obviamente la crisis económica desencadena conflictos políticos y sociales internos que son coadyuvados por presiones de trascendencia para los pueblos latinoamericanos: el uno la influencia de la revolución cubana de 1959; y el otro la concomitante acción de los E.U. mediante el Programa de Alianza para el Progreso en 1961, en el que el idearlo reformista, desarrollista se convierte para el continente en el evangelio económico político de los grupos dominantes (Baez René; 1977:250). Sus acciones más importantes se encaminharán al mejoramiento de los servicios al sector rural y la Ley de Reforma Agraria será uno de los argumentos para obtener justicia socialy que si bien no transforma la tenecia de la tierra, elimina ciertas formas feudales de supervivencia (Velasco, Fernando: 1981: 202).

La dependencia económica con los E.U. tiene otras caracteristicas:

-- acentuamiento de la brecha tecnológica

- penetración del capital extranjero a través del sector industrial.

Para el sector salud, este período es el dinámico por cuanto se siguen com los programas de erradicación de la malaria, que luego de la finalización de los servicios del SCISP en 1964, prosigue bajo auspicios del gobierno nacional y de los E. U., que da una contribución econòmica de 631 mil dólares para el programa en esta década (AID; 1972).

Por otro lado, la Misión Andina, impulsada desđe 1963 como programa nacional, viene a ser coherente con lo que sucede en el agro serrano en esos años. El carácter desarrollista del Estado, en cuyo modelo la Reforma Agraria viene a viabilizar la incorporación del campo serrano al sistema capitalista encuentra en la Misión Andina un ca- 
mino para apoyar esta iniciativa. La Misión Andina viene a ser un programa de desarrollo rural integral, que abarca aspectos de agricultura, vivienda, educación, caminos vecinales, mejoramiento del agua y de la salud, Financiado por un convenio suscrito entre el gobierno del Ecuador y el Banco de Desarrollo llamado "Integración del campesinado" en 1963 (Barsky, Osvaldo el al.; 1982:21), permite la creación de infraestructura para el desarrollo de las comunidades a las que va dirigido. Las comunidades beneficiadas son exclusivamente las del Callejón interandino y a las que nunca antes había llegado ningún servicio; comunidades, no ligadas al sistema hacendario, en las que los rasgos feudales y precapitalistas estan presentes.

De esta manera el Estado, con el apoyo da E.U. , emprenderá con la Reforma Agraria y con este programa que incorporará a ese campesino marginado al sistema, para lograr cumplir sus objetivos de desarrollo del capitalismo en las regiones más atrasadas. Parecería que el papel má importante de la Misión Andina fue penetrar en comunidades indigenas cerradas y viabilizar sus vinculaciones a las formas de producción capitalista a través de los servicios que ofrecía y que iban a modernizar su forma de vida.

La prestación de servicios de salud bajo este contexto es concebida como parte del desarrollo general, hecho que por primera vez se da en el país.

Además el Estado debe consolidarse como uma institución planificadora y ejecutora de una politica, crea no solo la Junta de Planificación ya mencionada, sino un Ministério de Salud Pública en 1967.

La mayoría de los servicios que dan salud continuan siendo los hospitales que dan asistencia curativa, con algunas modificaciones que son concomitantes a la modernización del Estado a través de la especialización médica lo que significa adquisición de equipamiento sofisticado, adopción de nuevas técnicas curativas quirúrgicas (operación de corazón abierto) etc.

La OPS reemplaza con su presencia e influencia al SCISP que parte en 1964 y a la Fundación Rockefeller, y es la encargada de fortalecer en nuestro país, la política emitida a nivel regional, en la Primeira Reunión de Ministros de Salud Pública para las Américas.

\section{La Educación de Enfermería}

Este período para enfermería se lo puede calificar como Cadernos de Saúde Pública, R.J., 2(4): 167-196, abr/jun, 1988. 
"transitorio" porque ocurren hechos que modifican la educación de enfermería del siguiente período. En el se gestan algunas de las instituciones cuya relación será significativa para la definición de la Primera Política de Educación de Enfermería del país promulgada en 1972.

Tal vez, como nunca antes, la relación entre las condiciones económicas sociales del país, la influencia internacional a través de las agencias norteamericanas y el desarrollo de enfermería se vea íntimamente ligados.

Para ejemplificar esta afirmación, detengámonos a analizar el principal acontecimiento sucitado en estos 10 años y que a nuestro modo de ver es la creación de una Facultad de Enfermería que ofrece la licenciatura.

Alianza para el Progreso a través de la USAID y el Punto IV, la Universidad de Saint Louis de Missouri, y la Pontificia Universitad Católica del Ecuador abren la Facultad de Enfermería en 1965, siendo esta organizada por 6 hermanas de la Caridad bajo la dirección de Sister Virginia Kingsbury, las mismas que luego son reemplazadas paulatinamente por personal ecuatoriano debidamente capacitado en los Estados Unidos.

Qué motiva a la Universidad Católica a abrir una Facultad de Enfermería? La evaluación que realiza Sister Kinsgburg en 1962, cuando por encargo de la Universidad de Saint Louis visita el Ecuador y en su informe explica las razones para abrir dicha Facultad: "1) Mal prestigio de la Enfermería en el país; 2) Condiciones de trabajo pobres; 3) sala. rios bajos y migración de enfermeras a otros países". Este hecho preparará el terreno para que enfermería sea una carrera reconocida como universitaria y no sólo profesional. Algunas escuelas dejan de ser "adscritas" a las universidades en la década siguiente para ser escuelas de una facultad de Ciencias Médicas con lo que comienzan a ser parte de los organismos universitarios.

La tendencia en la educación no sufre un cambio sustancial, a nuestro modo de ver permanece siendo nigyingeleana. A pesar del gran desarrolo de la Salud Publica al que arriban otros países latinoamericanos como Chile; $y$ de que se fundan varias escuelas de Salud Pública, aquella influencia no se deja sentir en la formación de nuestro recurso.

Es necesario destacar que si bien el sentido del curriculum 
persiste en lo curativo, incorpora un nuevo elemento: brinda atención al paciente como ser integral (1).

Para el desarrollo de la educación en Enfermería acontece otro hecho que también nace a finales de la década y provoca nuevas situaciones en 1970 a 1980 ; es la creación de la Asociación Ecuatoriana de Escuelas de Enfermería, ASEDEE, con la coaboración de las asesoras de la OPS/ OMS.

Esta creación no es un hecho fortuito, dos son los antecedentes. Por un lado hay una corriente latinoamericana impulsada desde el Primer Seminario de Educación Médica em Colombia en 1955, para formar instituciones que agrupen a las distintas, facultades y escuelas del área de salud. Jorge Andrade lo menciona en su trabajo "Marco Conceptual de la Educación Médica en América Latina" (2). Es pues, una orientación de esos años. Por otro lado, en nuestro país ya se ha formado la Asociacíon de Facultades de Medicina del Ecuador, AFEME, cuyo secretario ejecutivo, Miguel Marquéz, juega un papel importante como motivador de las enfermeras para la creación de ASEDEE, que inició sus actividades el 24 de agosto de 1969 y alcanzó su personería jurídica en 1972.

Para fines de la década cada escuela y facultad tienen las conclusiones y recomendaciones emanadas del Primer Seminario de Educación realizado el 24 de agosto de 1969 , las que de manera sintética gravitan en torno a las necesidades cuantitativas y cualitativas actuales y futuras del personal; a los niveles profesionales de enfermería que deben ser preparados y a las decisiones a tomarse en relación con el papel que corresponde al personal de enfermería y a las funciones de cada nivel. Los tres temas que preocupan a las escuelas tienen total relación con lo que está sucediendo en la prestación de servicios.

La escasa producción de recursos de enfermería puesto en evidencia en un estudio de investigación efectuado en 1953 por la Asociación Ecuatoriana de Enfermeras con la asesoria de la OPS/OMS y denominado "Estudio de Oferta y emanda del personal de Enfermería" demuestra la necesidad de incrementar el número de auxiliares de enfermería cuya formación sea de exclusiva competencia de enfermeras, lo cual se efectiviza mediante un decreto en 1974.

Para hablar de lo que sucede en la prestación de servicios de enfermería es necesario hacer referencia a algunos acon-

1 Según los lineamientos de la OPS en sus documentos para salud, el hombre se lo con. ceptualiza como un ser bio. sico-social y se maneja un concepto de salud-enfermedad biologista, no social. Posteriormente se profundizo este planteamiento..

2 Andrade dice: "Esta recomendación fue generalizada a los demás patses latinoamericanos en la Primera Conferencia de Facultades Latinoamericanas de Medicina reunida en México /UDUAL Posteriormente en 1960 en Montevideo se recomienda el establecimiento de la Federación Panamericana de Facultades y Escuelas de Mudicina, lo cual se hace en la Tercera Conferencia de Viña del Mar.

La fundación de FEPAFEN ha estado durante años sujeta a la polémica ya señalada como un caso de influencia de los E.U. en la. solución de los problemas eduactivos latinoa. mericanos" (Vol 12,M2, 1978; 151-152).

Cadernos de 'Saúde Pública, R.J., 2(4): 167-196, abr/jun, 1988. 
3 En la Carta de Punta del Este los gobiernos de las Américas programaron el desarrollo económico y social; de tal manera que se convirtió en "un instrumento jurldico para toda esta empresa continental". El Plan Decenal de Salud Pública de la Carto de Punta del Este sinvió de norma para la formulación de programas $y$ proyectos nacionales en el periodo 1962-1971. Su análisis fue objeto de las dos primeras Reuniones Especiales de Ministros de Salud de las Americas, que tivieron lugar, respectivamente, en abril de 1963 (Washington D.C.) $y$ en octubre de 1968 (Buenos Aires, Argentina). Los infor. mes finales (4,5) de estas reuniones contienen recomen. daciones, las que se incorpa ran a la politica de la OPS y de la OMS" (Plan Decenal de Salud para las Américas, OPS/OMS. Documento oficial No. $118 ; 73 ; 3)$. tecimientos que se van dando desde comienzos de la década. Así:

1. De la Primera Reunión de Ministros de Salud de Latino. america se deriva el compromiso del país para implementar un Plan Decenal de Salud Pública que sirva de normas para la formación de proyectos nacionales en el período 1962 a 1971 . Es por ello que en 1960 se elabora en el Ecuador al Primer Plan Decenal de Salud Pública cuyos objetivos deben cumplirse en 10 años (3).

2. El SCISP deja el país, pero salud no se queda huérfana de asesoria internacional. USAID en el documento por los "30 Años de Colaboración Económica entre Ecuador y E.U. " señala que existen varios organismos nacionales que colaboran en salud, etc., estarán colaborando con el gobierno ecuatoriano"; predicción que, efectivamente se cumple con más énfasis a partir de 1970.

3. En 1967 se crea el MSP siendo Ecuador el último país de la América en fundario. Hasta ese entonces fue el Ministerio de Provisión Social quien se responsailizó de salud a través de una Subsecretaría. Los hospitales existentes pertenecian en su totalidad, a excepción de los de la Seguridad Social, a la Asistencia Social, creada por Decreto Supremo a comienzos de sigilo por Eloy Alfaro; ésto trajo como consecuencia la ausencia de una política de salud francamente explícita. Para la década existian preponderantemente el Plan Decenal al que hicimos referencia.

4. La política latinoamericana en salud es la Extensión de Cobertura hacia el campo. Para cumplirla surge la necesidad de elaborar un Plan Nacional de Salud Rural, cuya programación es una de las primeras actividades del flamante Ministerio. De 1969 se lo formula con la colaboración de la OPS/OMS y la Junta Nacional de Planificación. Este lo aprueba el Congreso del año de 1969 y a partir de la siguiente década comienza a ser aplicado. (Castílo, et al; 1983).

Estas situaciones también de trasición, son las que marcan la práctica de enfermería, la que continua encerrada en los hospitales en su generalidad. A ellos comienzan a llegar aquellas enfermeras preparadas en las escuelas y que tienen, sobre todo, la influencia del SCISP. En el caso de Quito, su hospital popular, el Eugenio Espejo, es donde se nota dicha influencia reflejada en: aumento de personal de enfermeras seglares (el $50 \%$ es todavía personal religioso sin títulos universitarios), cambios en el Departamento de Enfermería con inicios de integración docente assistencial, conformación del Comité de Areas Cliínicas. De esta manera se pue. 
de apreciar las innovaciones que produce el SCISP en dicho hospital sin que se generalice para todos los hospitales del país, pues en ellos la atención de enfermería está en manos de las religiosas y mantiene la práctica las características nigtingeleanas.

\section{SEGUNDO PERIODO: 1970 - 1977}

La década del 70 evidencia la consolidación del moderno Estado ecuatoriano, que a través de la producción, explotación y comercialización del petróleo, se convertirá en receptor de la masa capital-dinero proveniente de él, dejando así de depender de la tributación de los productos agrícolas tradicionales controlados por la oligarquía.

El Estado pasa así a una fase de mayor intervencionismo, acentuandose su papel de dispositivo regulador y orientador de los distintos sectores de la economía en favor de la acumulación vía industrialización.

A partir de 1972 una dictadura militar de corte desarrollista $y$ a veces con ciertos matices progresistas promueve una política petrolera nacionalista, efectivización de la Reforma Agraria, fortalecimiento de instituciones estatales que llevan a la par el aumento desconmesura do de la burocracia.

La penetración del capital extranjero es notoria, éste invierte en la industria y trae importación de tecnologia. El desarrollo técnico y científico conileva una cresciente división del trabajo, que ne el área de salud se refleja en un incremento de la especialización de la atención médica y el surgimiento de las empresas médicas en la medicina privada en contraposición del tradicional consultorio privado.

Para esta época el Estado cuenta con más recursos que en la década precedente para impulsar planes, ya que la renta proveniente del petróleo nos habla de bonanza a diferencia de la crisis económica que aparejaba el desarrollismo de los años 60 .

Es así como las obras de infraestructura emprendidas por el Estado reportan para salud la creación de 25 hospitales centro de salud y 300 subcentros de salud, en cabeceras cantonales y parroquiales respectivamente.

Así mismo el tinte centralizador del Estado en salud se refleja a través de medidas concretas como: la supresión de algunas entidades autónomas de salud: la Liga Antituber- 


\section{ARTIGO}

4 Las aspirantes a la ENE eran seleccionadas tras un examen que tomaba en cuenta no solo los requisitos educativos sino modales, costumbres, buena presencia. No hay que olvidar que dentro de la educación de la ENE era muy importante enseñar a: comer, vestirse, caminar, dirigirse a los superiores, hablar correctamente, ser puntuales, es decer dar la imagen de una "legión de angels blancos dentro de un régimen de internado tipo norteamericano flexneriano.

5 Es clave el proceso que sufren los escuelas de enfermerta para formar parte de unidades académicas (Facultades de Ciencias Médicas o de Salud).

En las Universidades Estatales, si hacemos una cronología, las escuelas se incertam a comienzos de la década del 70. $E l$ orden cronologica seria el sigulente:

1970: ENE

1973: Escuela de Enfermería San Vicente de Paúl de la Universidad Católica de Santiago de Guayaquil.

1973: Escuela de Enfermeria de la Universidad de Guayaquil.

1977: Escuela de Enfermeria de la Universidad Técnica de Manabí. Esta nació adscrita a la Facultad de Ciencias Sociales y Educación. en junio de 1977. Tres meses depúes es parte de la Facultad de Ciencias de la Salud.

Las siguientes Escuelas nacieron como entidades universitarios:

1965: Facultad de Enfermerta de La PUCE

1968: Escuela de Enfermerta de la Universidad de Cuenca

1976: Escuela de Enjermerta de la Universidad de Loja

1980: Escuela de Enfermerta de Manta, extensión de la Universidad Laica de Guayaquil.

1982: Escuela de Enfermería de la Universidad Técnica de Machala. cula (LEA), Solca, gran parte de los hospitales de la Asistencia Pública,centros de salud municipales, Misión Andina que pasa a ser parte de los programas del MSP.

La planificación a la que hemos hecho referencia, también está inmersa en salud con planes quinquenales, evaluaciones y reformulaciones que se producen en este perfodo: el Plan Nacional de Salud Rural, en vigencia desde 1970; el Plan Quinquenal 1973-1977; el Plan País 1974-1977.

Sin embargo, la evaluación de algunos de estos planes, efectuada, por el mismo Estado, no arroja mayor modificación en los cuadros de salud de la población (Suárez, José y López, Raúl, Evaluación de Plan Nacional de Salud Rural, p. 35).

\section{La Educación de Enfermería}

También en el ámbito de la Universidad se van a operar modificaciones producto, en este caso, de la II Reforma Universitaria impulsada por el Dr. Manuel Agustín Aguirre, a finales de la década precedente.

Dos elementos se deben destacar de los postulados de esa nueva Universidad que se trata de construir, y que impactarán en enfermería: Por un lado, se trata de la apertura de la entidad a través del libre ingreso, que hecha al olvido el elitismo de algunas Escuelas de Enfermería tales como la Nacional (4), y por otro lado, el reconocimiento de todas las Escuelas como partes integrantes de la Universidad, dejando de ser tan solo adscritas(5).

De esta manera, Enfermería, a mediados de la década del 70 completa lo que poderíamos llamar la profesionalización universitaria de la carrera al ser parte de la Universidad Ecuatoriana y al tener bajo su rresponsabilidad la formación de todo el recurso de Enfermeria, sea éste profesional universitario o auxiliar de enfermería.

\section{La influencia de la OPS en la Educación de Enfermería}

La OPS estará presente en estos 7 años no solo en enfermería, sino en salud, asesorando los planes y programas del MSP. "La OPS teniendo como base nas directrices del Plan Decenal de Salud Pública de la Carta de Punta del Este, ha comenzado a formular su política, programas y proyectos para el período 1962-1971" (Plan Decenal; 1972). De esta manera, si en los años anteriores determinadas Misiones llegaronal país, hoy la OPS es la agencia que de una manera 
distinta orienta la política de salud (no solo del Ecuador, sino de los paises de Amérnica Latina comprometidos en las reuniones de Ministros de Salud de las Américas) a desplegar lineamientos uniformes para elevar el nível de vida y de salud para las Américas. De esta forma el Primer Plan Decenal de Salud, por ejemplo enfatiza en la programación y planeamiento de salud en cada país (1962-1972). En enfermería se promueve el planeamiento nacional para lo cual se hace un levantamiento de recursos existentes.

Esto ya se había realizado antes en 1963 cuando se efectúa el primer estudio, auspiciado por la OPS (6).

El Segundo Plan Decenal (1972-1982), se plantea como meto elevar la esperanza de vida mediante la Extensión de Cobertura de servicios de salud a la población no atendida. En cuanto a enfermería busca acelerar la producción del recurso y la integración docente-asistencial.

De igual manera en la educación de enfermería, la presencia de las asesoras de la OPS es de relevancia para las reformas que se introducen. En consecuencia, dichas reformas también incluyen nuevas conceptualizaciones de salud y de práctica de enfermería aunque dentro del marco curativo, pero de acuerdo con las políticas de los Planes Decenales.

La OPS trabajando en intima relación con al organismo que agrupa a todas las Escuelas y Facultades de Enfermería, ASEDEE, y a través de los seminarios de educación que promueve, dará un gran apoyo y vertirá su influencia en la educación de estos años. La temática de los Seminários nos van a ejemplificar esta relación, que por cierto también es estrecha con los lineamientos del MSP:

- Necesidades Cuantitativas presentes y futuras del personal de Enfermería para los servicios de Salud del Ecuador, 1969.

- Oferta y Demanda del Personal de Enfermería del Ecuador, 1971.

- Formación de Auxiliares de Enfermería del Ecuardo, 1972.

- Integración Docencia servicio para la formación de la Enfermera que el Ecuador: necesita. 1973.

- Evalución del Año de Salud Rural de las egresadas de Enfermería en el. Ecuador: promociones 1973-1974, 1974.

Todos ellos fueron efectuados de 1969 a 1974 con el auspicio, financiamiento y asesoría de la OPS/OMS.

Cadernos de Saúde Pública, R.J., 2(4): 167-196, abr/jun, 1988.
6 Segun De Almeida Souza "el estudio de recursos de enfermerta fue una próctica de estrategia norteamericana recomendada para la America Latina, esto no solo para justificar cuotas de crédito sino también para planiffcar. . . lo que demuestra la permanen. cia de la influencia norteamericana en la región a través de la OPS" (1982). Traducción realizado por los autoras. 
CUADRO № 1

Concentrado de la Asesoria Ofrecida por la OPS/OMS a ASEDEE en los 14 años de vida del organismo 1969-1983

\begin{tabular}{lccc}
\hline \multicolumn{2}{l}{ PERIODO DIRECTORIO } & \multicolumn{2}{c}{ TIEMPO } \\
ASEDEE & No DE ASESORAS & As. Cortas & As. Largas \\
\hline $1969-1971$ & 4 & 4 & 1 \\
$1971-1973$ & 4 & 3 & \\
$1973-1975$ & 1 & 1 & \\
$1975-1977$ & 4 & 4 & 1 \\
$1977-1979$ & $1^{*}$ & 2 & \\
$1979-1981$ & 3 & 3 & 2 \\
$1981-1983$ & 3 & 17 & 2 \\
\hline TOTAL & 19 & 17
\end{tabular}

* En este período aún permanece Rosa Pedrós hasta 1978. FLUENTE: Archivos ASEDEE, 1969-1983.

ELABORACIÓN : Autoras.

De las 19 asesoras que ilegan en los 14 años de vida de ASEDEE, 13 lo hacen en los primeros años de la década del 70 , época en la cual se puede hablar de una tríada de relación entre ASEDEE, la OPS y el MSP. Esto lo afirmamos porque Enfermería en el MSP se fortalece cuando en 1972 $a^{*}$ raíz de la organización del Ministerio, se crea el departamento nacional de enfermería con una estructura técnicoadministrativa concordante con el sistema regionalizado de servicios contemplado en el Plan Nacional de Salud. Años más tarde, como reconocimiento de este subsistema, mediante acuerdo ministerial se conformaría la División Nacional de Enfermería. Esta División promulga una Política Nacional de Enfermería que recoge los planteamientos del Estado respecto a salud. Es ASEDEE quien está directamente involucrada en la organización de estas instancias que son de relevancia para Enfermería.

Para terminar el período las asesoras de la OPS llegan para impulsar un Centro de Tecnologia Educativa que va a funcionar em la ENE. La OPS na generado la creación de un Centro Latinoamericano de Tecnologia Educativa en Rio de Janeiro llamado NUTES/CLATES, el mismo que en 1974 invita a la ENE a participar en el Proyecto.

\section{La Primera Política de Educación de Enfermería}

ASEDEE en su tercera reunión de Junta Directiva de 1972 se plantea la realización de una Política de Educación que oriente la formación del recurso, ésta entrará en vigencia a finales do año. 


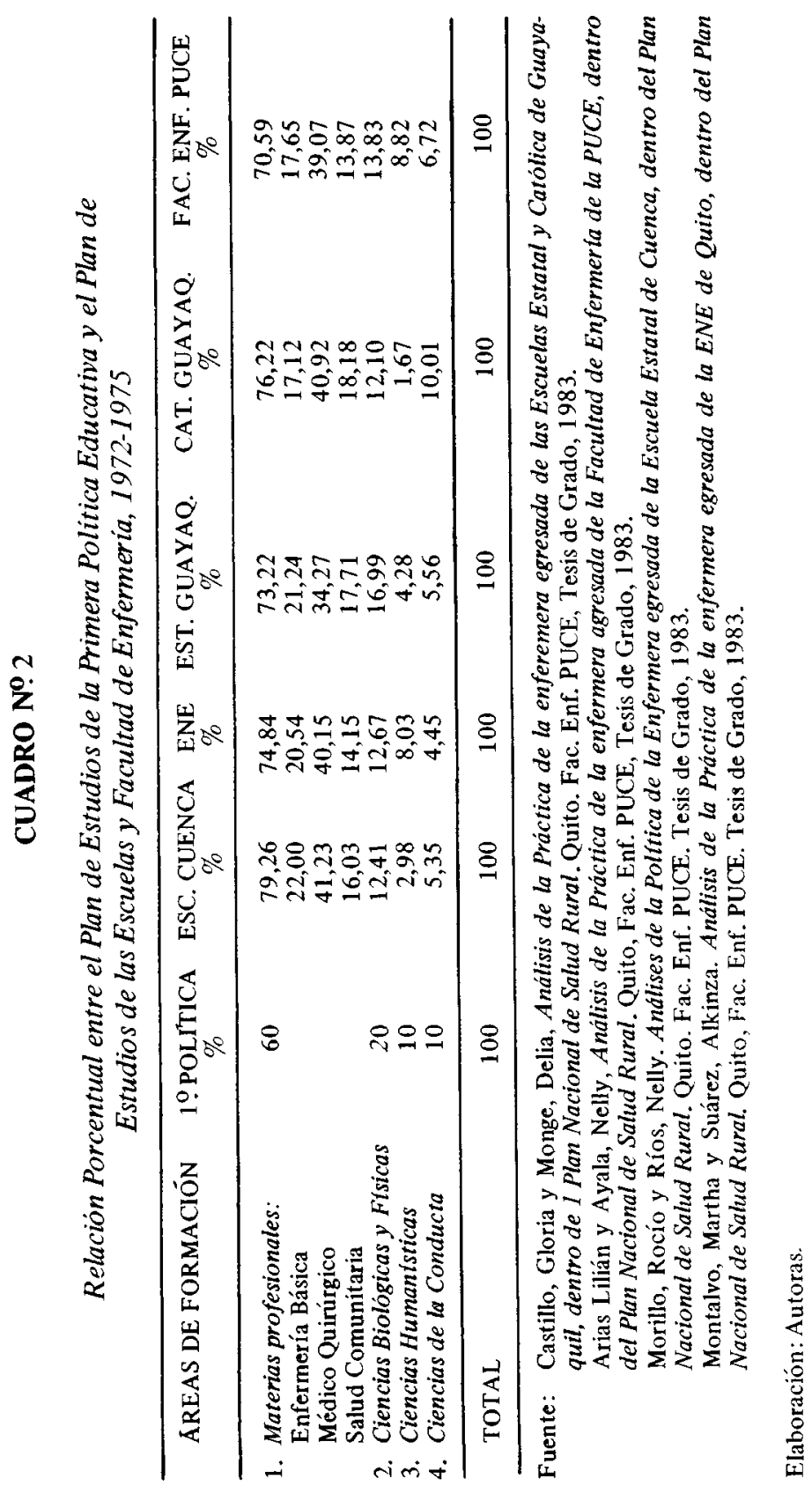


Lo más relevante de esta política es la unificación de planes de estudio que propone y que logran plasmarse an las cinco escuelas de enfermería que en ese momento funcionam en el país. En el cuadro 2, podemos apreciar um consolidado que demuestra cómo las diferencias entre los planes de las cinco escuelas son poco relevantes y se refierem en cuanto a cargas horarias, manteniendo todas el mismo esquemas de materiais y contenidos.

Por otro lado, en cuanto a la formación de recursos, la política responderá a las necesidades del Estado ecuatoriano en lo que a Extensión de Cobertura se refiere, a través del incremento del número de auxiliares de enfermería sobre el de enfermeras. Basta citar que a partir de esta política la relación entre metas propuestas y alcanzadas en cuanto al número de enfermeras formadas, no logra pasar el $80 \%$ (817); mientras que en lo que se refiere al personal auxiliar de enfermería, la meta es rebasada, llegando al $14 \%$ (Propuestas: 2.780; alcanzado; 3.897) (Datos extraídos de: De la Torre, Patricia y Velasco A. Margarita)

Esto reflejará el interés del propio MSP de incrementar el número de personal, a menor costo, sin importar la calidad, buscando únicamente cubrir con personal las instancias creadas dentro de la política de Extensión de Cobertura. A instancias del MSP las Escuelas y Facultad también dejarán de formar enfermeras en 4 años para hacerlo en 3. Cabe señalar, que a pesar de este apoyo de las Escuelas y Facultad, el personal formado continuó trabajando en la zona urbana en hospitales y muy pocas en la rural, sin llegar a la población rural dispersa.

La crítica principal que se puede hacer a esta política es la ausência de referenciales teóricos explícitos que clarifiquen el papel de ese recurso a formarse dentro del ámbito de una sociedad concreta, la ecuatoriana dividida en clases sociales, con una población pobre, marginal, que cada vez es mayor, con un perfil epidemiológico típico de país subdesarrollado. Es decir de país sujeto a una dependencia económica externa, con una economía insegura, que a ratos provoca crisis social; con un sistema de salud endeble y que no es único; con un presupuesto para salud que alcanza para pagar salarios y no para desarrollar programas, etc, etc. No parte del piso sobre el cual estamos parados, tal vez como reflejo de la influencia de los Planes Decenales, en lo que se conceptualiza y se uniforma políticas de salud a nivel latinoamericano, $y$ a las cuales se encuentram muy ligados las Escuelas y Facultad de Enfermería. El marco teórico que se maneja implícitamente en la Primera Polí- 
tica se deriva de esto, no de nuestra realidad, y más adelante nos centraremos a analizar la tendencia en la educación que esto trae implícito.

Nuestra segunda afirmación se refiere al momento del desarrollo de una práctica de enfermería, en la que al componente del Estado es preponderante. Esto amerita una reflexión: el cuerpo doctrinario de la educación de Enfermer ía no nace de un pensamiento universitario, que independientemente del Estado, sea la conciencia crítica de la sociedad, como lo manifiesta la II Reforma Universitaria. No. Para 1972, las escuelas apenas han sido trasplantadas del seno del Estado ecuatoriano, que las acunó desde su fundación, con la ayuda de las organizaciones internacionales: Rockefeller, $\mathrm{SCI}$ y AID, a una nueva tierra en la que deben sentar raíces, haciéndose un lugar; este espacio es la universidad.

A partir de esta nueva instancia, las respuestas que la educación de Enfermería debe dar, no son las de número de recursos humanos únicamente, sino de tipo profesional, coherente con las necesidades de salud de la población. La Primera Política no asume este desafio, le contesta al Estado, le es funcional a sus necesidades, que muchas veces no son el reflejo de lo que requiere la población. La polí. tica no aclara lo que entiende por Estado, ni por "sistema de salud", del cual dice ser parte, sin declarar su pertenen. cia a la universidad, y por ende, su autonomía con respecto al Estado. Mecho que en el análisis de la Primera Política, que hacen las Escuelas y Facultad en 1979, es uno de los puntos primordiales. La mayoría de allas critica que la Política es atentatoria a la autonomía universitaria.. El paso del tiempo, el cambio en la relación ASEDEE-MSP, irá demonstrando a la educación de enfermería que esa participación se va modificando y en momentos se vuelve con. tradictorla(7). Hasta que la educación de enfermería asuma ese nuevo punto de partida, pasarán años, los necesarios para convertir sus objetivos en los de la universidad. Mas, sería injusto y parcial dejar algunos aspectos sueltos en nuestro análisis. La educación de enfermería en 1972 comenzó otra etapa en su vida dentro de la universidad, pero su influencia no podía darse inmediatamente. Debemos partir del princípio que los fenómenos en la realidad se dan como procesos y no como fórmulas o recetas y eso nos permitirá analizar de una manera objetiva, no bajo la óptica del bien o del mal. Eso permite apreciar en la medida justa lo que sucedió en el pasado y obtener sabiduría para el futuro.

La educación de enfermería pasa por ese momento que se refleja en la Primera Política, porque era coherente y nece- 
saria para su crecimiento. No se podería pedir más, cuando el mismo desarrollo de las ciencias sociales y la interpretación socio-política-antropológica y económica del país, estaba comenzando a dar sus primeiros productos (Alejandro, Moreano; $1981: 1-20$ ).

Por tanto, las docentes que participan en su formulación traen un bagaje que o producto de su práctica hospitalaria o de salud pública, inmersa siempre en el marco del Estado de dónde se generaría entonces una visión más global y crítica de lo que habla que hacer: sus efectos, sus respercursiones a largo prazo. Algunas deestas docentes, en los años siguientes, pudieron desarrolar su formación hacia otras lineas de pensamiento.

\section{La tendencia de la educación de Enfermería}

Como hemos señalado, una nueva tendencia se hace presen. te en la politica de salud, y naturalmente, en la formación del recurso de Enfermería. Dentro de el la enfermería crea un sistema de valores que no es producto de la Enfermería ecuatoriana, sino que se convierte en una corriente latino. americana difundida a través de Seminarios patrocinados por la OPS/OMS. Este sistema al que hacemos referencia tiene como base la consideración de la enfermedad como um episodio de la vida del hombre como ser bio-psico-social "en equilibrio dinámico con su medio ambiente y considerando las modificaciones del medio, tanto interno como externo, que pueden alterar dicho equilibrio. Las acciones de salud en las etapas de prevención primaria, secundaria y terciaria en las que interviene la enfermera mediante la aplicación de sus conocimientos científicos y los de su profesión, ayuderán al hombre a mantener o a establecer su equilibrio" (OPS/OMS, 1971).

Esta cita es el marco conceptual sobre el cual se desarrolla la formación de recursos de enfermería a partir de 1972 , cuando se promulga la Primera Política de Educación La referencia que presentamos es del informa Final del Seminario sobre Enseñanza de Enfermería a nilve universitario de la OPS realizado entre marzo y agosto de 1971.

Como se liga esto a la Primera Política de Educación, si ella no explica las corrientes del pensamiento que van a orientarla. Cuando ella enuncia las funciones de la enfermera y la auxiliar éstas son las mismas que el Comité de Expertos de la OPS/OMS recomienda, luego del Seminario de Educación de Enfermería de Lima, auspiciado por la OPS/OMS y que sirve como fuente de referencia a la Pri- 
mera Política. En este aparece además de lo ya citado, la metodología de trabajo de enfermer ía que recibe el nombre de "proceso de Enfermería".

Lo interesante es descubrir qué ubyace detrás de este concepto de salud-enfermedad del proceso de enfermería.

El hombre, tal como lo describe el planteamiento que hemos traído aqui, tiene un comienzo y un fin biologista. Cumple un ciclo vital y punto. No se percibe su ser social, ni su relación con el conglomerado humano y su reflejo en su salud. Esta es una concepción propia del positivismo. Es una captación fenomenológica, ecologista e individualista. En el fondo, lo que se esgrime es una visión idealista del mundo. Es decir, de aquel punto de vista que sostiene que las cosas son estáticas. Los hechos del universo, sus formas y sus especies, son aislados unos de otros y eternamente inmutables.

Esto se liga efectivamente a la práctica hospitalaria en la que el mundo se desenvuelve fuera de las paredes del hospital. Adentro de él no existe el problema trabajo-enfermedad, desigual distribución del ingreso, desocupación, diferencias étnicas y culturales, problemas de saneamiento ambiental. En el hospital se trata el desequilibrio-enfermedad, se trata de "ayudar al hombre a restaurar éste, acelerando su retorno al bienestar físico y emocional". Porque la salud, bajo esta óptica, es un hecho aislado, no es consecuencia de un proceso dinámico, sino de una causa con un solo efecto. Es lo contrario a comprender a la salud como parte y consecuencia de la problemática social, cultural, económica y política de los pueblos. No es nada más que ese desequillbrio que tiene como causas físicas o emocionales, pero no sociales.

En cuento a la práctica de salud comunitaria, ese conceptualizar a la enfermedad como la ruptura de un equilibrio, se quedará buscando soluciones inmediatas, unilaterales, que pronto son absorbidas por una realidad que es mucho más compleja y a la que los paliativos hacen poca ingerencia, y a la final no la terminan cambiando.

En cuanto al proceso de enfermería, al igual que el método de resolución de problemas, recogido también por la Primera Política de Educación, es asumido como um método propio, característico de enfermería y que es la aplicación del método científico. Esto merece una crítica, respecto a las diferencias de procedimientos que hay entre uno y otro, y que sería lo que precisamente los diferencian.

Cadernos de Saúde Pública, R.J., 2(4): 167-196, abr/jun, 1988. 
Hemos dicho que el proceso de enfermeria se asienta sobre una concepción fenomenológica de la realidad. Un idealista como Mario Bunge, que comparte esa misma idelogia, plantea diferencias sustanciales para el método científico que se rige de las siguientes reglas:

1) Formular el problema con precisión y al principio, específicamente.

2) Proponer conjeturas bien definidas y fundadas de algún modo y no suposiciones que no comprometen en concreto ni tampoco ocurrencias sin fundamento visible. Estas son las hipótesis.

3) Someter la hipótesis a constatación dura y no laxa.

4) No declarar verdadera una hipótesis satisfactoriamente confirmada, considerarla en el mejor de los casos como parcialmente verdadera.

5) Preguntarse por qué la respuesta es como es y no de otra manera.

Estas reglas son universales. . . La aplicación del método científico son aplicaciones a la verdad. . . Las reglas deben estar basadas o compatibles con las leyes de la lógica. . El método científico es único, si sabemos que la ciencia es una disciplina que utiliza el método científico con la finalidad de hallar estructuras generales (leyes). .. El método científico es un modo de tratar problemas intelectuales, no cosas, instrumentos ni hombres, se usa para el campo del conocimiento" (Bunge, Mario, 1983:25-27).

Vemos pues, que ni siquiera comparado con pensadores de la misma línea de pensamiento idealista, los dosprocesosson iguales, ya que el proceso de enfermería trata deseguir los pasos del método científico aplicandolo a una práctica en la que se utiliza precisamente hombres e instrumentos y no problemas del pensamiento en el sentido filosófico-abstracto. Es decir que este proceso que se lo llama "propio" de enfermería, al integraria de manera incompleta a su práctica, lo aleja del método científico. El método científico es único para la ciencia, no se puede crear uno exclusivo para enfermería, a riesgo de que sea un seudométdo o una negación de él.

Y si traemos a colación otras partes del documento de la OPS/OMS, concluiremos que cada vez más éste se aleja de lo que es el método científico.

“...el proceso de enfermería es el método científico, pero aplicado con menos rigor" (OPS/OMS, 1980: 87). 
Una de las características de la ciencia es la utilización del método con absoluto rigor.

En el proceso de enfermería se aplica conocimientos científicos mas no se genera conocimientos científicos. El método específico de la ciencia. El proceso de enfermería es "un método" específico para organizar una práctica. En el proceso da enfermería se supone que "la obtención de impresiones y presentimientos y definiciones de Enfermería de problemas concretos a base de hechos y lo que puede significar" (OPS/OMS, Ibid) son equivalentes a las hipótesis del método científico, el cual niega las especulaciones, y se basa en supuestos objetivos, es decir, todo lo contrario de lo que son las impresiones y presentimientos. "El método científico defiende, que los hechos registrables a través de los órganos de los sentidos - sus datos no son sino el nival aparente, fenoménico de la realidad, no son más que efectos de procesos, determinantes profundamente interrelacionados" (CEAS, 1984:7). Esto es todo lo contrario a la concepción fenomenologica que aisla hechos; como que "ellos hablaran por su propia boca. Bajo semejante planteamiento, el método se transforma en una especie de recetario para la mezcla de ingredientes y la realidad no viene a ser sino la suma de combinaciones de "hechos" aparenciales o fenoménicos que se presentan en la superficie a simple vista. El método científico afirma que no se trata únicamente de medir o registrar un fenómeno, sino que tiene, además, que buscarse expresión formal de su contenido esencial. Esta profundización está r regida por las leyes que rigen el devenir del objeto estudio inmerso en la globalidad social" (CEAS, Ibid).

Estos dos aspectos: la concepción idealista y el proceso de enfermería como método científico, que hemos analiza. do aqui, probablemente sean una de las causas para que el recurso de enfermería no haya podido caracterizarse por su dinamismo, su criticismo y su posición de lucha.

Para enfermería, que as una profesión como las otras, la aplicación del método científico, no de un método específico, que no es el científico, le servirá para entender no sólo el desarrollo técnico de sus funciones sino el papel de la salud en la sociedad, de sus leyes y de esta manera poderá entender la realidad del país, no para verla a través de una vitrina, sino para dar soluciones, para transformarla. Sólo alli hablaremos de que formamos enfermeras críticas, sin conocimientos fraccionados y tecnicistas.

Con la vinculación entre una nueva concepción de la salud-

Cadernos de Saúde Pública, R.J., 2(4): 167-196, abr/jun, 1988. 
enfermedad y la adopción de una metodología de trabajo, la educación de enfermería podrá redefinir su compromiso social como carrera universitaria, lo que impactará no sólo en el mejoramiento de la calidad de los servicios de salud, sino en el cambio sustancial de las condiciones de vida de la población.

\section{TERCER PERIODO: 1977-1983}

Éstos 6 años coinciden con hechos fundamentales para la sociedad ecuatoriana. A partir de 1979 se inaugura el período democrático, 7 años de dictadura militar, Jaime Roldós Aguilera y Osvaldo Hurtado Larrea asumen el poder con el compromiso de crear "un nuevo Ecuador democrático, justo, humano y libre" (Plan Nacional de Desarrollo, 1979). Pero, al poco tiempo, el gobierno de marcado tinte populista empieza a soportar los síntomas de una de las más graves crisis económicas de la historia. A decir de Sohel Riffka, "La más aguda, sólo comparable a la gran depresión de los años 30" (Sohel, Riffka, 1983).

La aguda crisis se desencadena paulatinamente com déficits de la balanza de pagos, baja de las exportaciones de petróleo, devaluación de la moneda, elevación del cambio del dólar, acrecentamiento del endeudamiento externo al Fondo Monetario Internacional y crisis en la pequeña industria.

Para añadir un elementomás a este cuadro, el país soporta en el período 1982-1983 el măs agudo y avasallador invierno en la Costa que provoca inundaciones, pérdida de cosechas y miseria. La política económica del gobierno no encuentra salida a esta situación en la que el descontento social es común, con el consiguiente agravamiento de las condiciones de vida y de seguridad de las masas.

El Estado ecuatoriano, a pesar de la crisis se mantiene en su proceso de modificación apoyando al sector industrial. Las acciones del gobierno continúan enfocandose al agro. Así se crea una Secretaría de Desarrollo Rural Integral (SEDRI), encargada de ejecutar 17 proyectos en todo el Ecuador, que abarca a las 4 regiones naturales del país: Costa, Sierra, Oriente y Archipiélago de Gálápagos. De éstos, 5 están en ejecución; se incorporan más comunidades al seguro social campesino del Instituto Ecuatoriano de Seguridad Social (IESS).

La dependencia económica se evidencía a través de la deuda externa, y la crisis al interior del país es también reflejo de la crisis que sufre todo el sistema capitalista mundial.

Cadernos de Saúde Pública, R.J., 2(4): 167-196, abr/jun, 1988. 
El Estado pretende recuperar una imagen nacional en la que no calzan ya los asesores que como en el caso de salud y en los primeros años, asumen directamente la orientación de los programas (verbi gracia el norteamericano LLoyd director de La Sanidad), sino que hoy los programas de cooperación técnica se manejan a través de las instituciones ya consolidadas con las que cuenta el Estado. El Ecuador además, y coherente con la seguridad que parece imprimir el Estado nacional, busca alternativas al problema económico conjuntamente con otras naciones latinoamericanas para hacer frente de manera distinta que en las décadas precedentes, a la dependencia y a la misma crisis del capital.

Es palpable el empobrecimiento que sufre el Estado lo que para salud significa reducción de presupuesto y un freno a los programas de desarrollo. Es así como la asignación del Estado, alcanza para pagar salarios a una burocracia que desde la creación del MSP se ha acrecentado.

Los problemas de salud de la población se abordan desde el punto de vista curativo y desde el punto de vista preventivo, emprendiendo programas que bajen las casas de morbimortalidad, dando prioridad al binomio madre-niño.

Sin embargo, la recesión económica que hemos mencionado, convierte a ese servicio del Estado en un hecho poco impactante.

\section{La nueva tendencia en salud}

$\mathrm{Si}$ bien el Estado ecuatoriano inicia desde los años 60 y con más ímpetu en los 70 programas que tienen que ver con la recuperación, promoción y protección de la salud de vastos sectores de la población sobre todo campesina, no es sino a partir de la Conferencia Internacionla de Alma Ata, de 1978, cuando la salud comunitaria va a adquirir mayor preponderancia sobre todo en el plano de la discusión teórica y del discurso que el MSP maneja para definir sus acciones. Esto naturalmente influirá en la formación del recurso limitándose sin embargo, al ámbito de Enfermería.

La estrategia de Atención Primaria de Salud promovida por la OMS pretenderá lograr salud para tods en el año 2 mil y será parte de una política implementada a nivel mundial. De esa manera el país se verá envuelto en ella, ya no como en los años anteriores con orientaciones regionales a través de la OPS, sino con una consigna que a rebasado latinoamérica y que se ha convertido, a larga, en una respuesta de los pai-

Cadernos de Saúde Pública, R.J., 2(4): 167-196, abr/jun, 1988. 
ses centrales a la problemática de los países tercer mundistas.

El MSP pues, continuará su política de Extensión de Cobertura emanada de los Planes Decenales de Salud para las Américas, con la nueva estrategia de Atención Primaria de Salud (APS), la misma que pasará por una serie de modificaciones hasta tratar de plasmarla a la realidad ecuatoriana.

Así, el enunciado de APS emitido en la conferencia de Alma Ata irá siendo cuestionado, adaptado y ajustado, El concepto que dice textualmente:

"Atención Primaria es la asistencia sanitaria esencial basada en métodos y tecnologías prácticas, científicámente fundadas y socialmente aceptables, puesta al alcance de todos los individuos y familias de la comunidad mediante su plena participación y a un costo que la comunidad y al país puedan soportar, en todas y cada una de las etapas de su desarrollo con un espiritu de autorresponsabilidad y autodeterminación. La atención primaria forma parte integrante tanto del sistema nacional de salud, del que constituye la función central y el núcleo principal, como desarrollo social y económico global de la comunidad con el sistema nacional de salud, llevando la más cerca posible la atención de salud al lugar donde residen y trabajan las persones y constituye el primer elemento de un proceso permanente de asistencia sanitaria" (Informe de la Conferencia Internacional sobre APS, 1978)

Entre las críticas que ha sido objeto este concepto se menciona el hecho de partir de la sociedad como un fenómeno ahis tórico, innamovible, porque sus caractéres, sus problemas en fin ella misma, siempre ha sido así y por lo tanto siempre seguirá siendo asi en mayor o menor grado. Esta no toma a la salud como producto de las condiciones de vida, como expresión de al estructura social, sino como unasituación ajena, que se impone desde afuera, como algo aislado, desligado del hecho social. Esto descubre el sustrato de fondo del enunciado de APS: mejorar no cambiar la sociedad, para lo cual uno de los aspectos paliativos es salud, o sino de sostendrá ingenuamente la premisa de que la situación de salud cambiará y mejorarará la economía, esto lleva a plantear soluciones superestructurales a problemas estructurales que no son atendidos en el texto de la declaración. 
En esa medida el programa de APS se vuelve inorgánico ya que no está en conexión con otros aspectos de fondo. Se generaliza el problema de salud a todas las sociedades y países sin discriminar diferencias radicales. No discrimina el problema de salud tomando en cuenta diferencias económicas, políticas, culturales fundamentalmente geograficas. étcnicas; inclusive conlleva intrínsicamente el supuesto falaz de querer convertise en un programa modelo, único, aplicable y realizable por igual y por encima de las diferencias en todas partes: ésto convierte el proyecto en un proyecto abstracto. El propio documento hace depender el éxito del programa a la situación económica del lugar y de la comunidad con lo cual justifica com anterioridad el fracaso para aquellas zonas donde no se puede llevar adelante por falta de recursos, com lo cual se mantendrá vigente la situación actual desigualdad con respecto a la salud. (Nelson Reas$\cos ; 1982: 1-2)$.

Al Ecuador parece que la política en mención, le produjo algunas dificultades en su aplicabilidad, esto lo demuestra la evolución que sufre su conceptualización y por ende su operacionalización.

Para 1977 al MSP ya toma en cuenta la Atención Primaria en sus programas, estrategia que un año más tarde será oficializada a nivel mundial en la Conferencia ya mencionada. Y en este año se entiende a la APS como:

"Una estrategia para la extensión de cobertura, que se basea en la utilización de tecnologias eficientes, simpples, sencillas o combinación de tecnologías para la solución de las necesidades básicas de salud que son las más frecuentes en la comunidad. (MSP; 1977:11).

Para ese momento, la APS aparece como una necesidad imperiosa de readecuar tecnologías, considerándola como uno de los aspectos importantes en la solución de la problemática de salud.

En 1980 el concepto sufre una notable variación. Esto es lo que se lee en uno de los Manuales de uso Institucional del MSP:

“Atención Primaria de Salud con participación comunitaria, significa la satisfacción de las necesidades prioritarias de salud de las familias más expuestas a riesgos, utilizando sus propios recursos, reconociendo como ejecutor de las acciones a un miembro de la 
comunidad, bajo condiciones que permitan la integración del sistema informal al desarrollo de acciones integrales de salud debidamente apoyadas por el sistema formal dentro de la adecuada regionalización de servicios" (MSP-FODERUMA; 1980: 16).

En el informe final del Seminario de Revisión de los Manuales de APS realizado em 1981, la definición es:

"Atención Primaria de Salud es la acción que busca la satisfacción de las necesidades de salud más frecuentes y prioritarias de toda la población y promueve su desarrollo integral mediante la participación conjunta y coordinada del equipo de salud institucional, de otras instituciones de desarrollo y de la comunidad organizada y capacitada" (MSP, $1981: 2$ ).

Bajo la luz de estos conceptos es posible reflexionar sobre cómo se ha implementado APS en nuestro país. Los tres conceptos evidencian la evolución operada por el MSP en el afán de adecuarla a la realidad ecuatoriana.

El programa de APS es en la práctica centralizado en la formación de promotores de salud, que en la mayoria de casos son líderes de las comunidades reclatos por el MSP de acuerdo e una serie de pautas. Los promotores se capacitan en cursos de un mas dictados por enfermeras.

El MSP, a través de las auxiliares de enfermería de los puestos minimos supervisa las acciones de los promotores. El se convierte en el miembro de la comunidad que enlaza el sistema de salud formal-regionalizado, a los pacientes que requieren de atención más compleja que la aportada por dicho promotor en el primer nível.

8 En la investigación sobre APS de las Agencias no Guberna. mentales de Salud, realizado por el ININMS em 1980, se destacó que la mayoria de pro. motores haclan uso indiscrimi. nado de medicamentos. A manera de ejemplo: so constató que en la parroquia Columbe. Provincia del Chimborazo, algunos de los promotores de sa. lud del Subcentro habian colo. cado pequeños botiquines en sus casas, cuyos medicamien. tos fueron adquiridos en las boticas de Riobamba.
En la practica este criterio de interpretación de APS, trae algunas consecuencias que no benefician a la politica, sino más bien la cuestionan:

La supervisión no se da por la escacez del recurso de enfermaría en las áreas rurales. El promotor capacitado para fomentar acciones de prevención, en muchos casos pone más énfasis en lo curativo, con exagerado manejo de medicamentos (8). El promotor recibe una bonificación del Estado (MSP-FODERUMA), se convierte así en un asalariado, connotación no observada en la declaración de APS y que provoca algunos conflictos al trastocarse el sentido de trabajo comunitario de reciprocidad al de trabajo asalariado 
y a la postre él termina de personaje híbrido al no ser parte ni del sistema formal de salud ni de su misma comunidad.

Por último, la referencia no se logra al no existir en la práctica um sistema regionalizado de servicios. La regionaliza. ción recién se esta tratando de consolidar para acabar con la irracionalidad de un sistema cuya caracteristica es el desperdicio del recurso humano y económico por la falta de organización. Este elemento costo beneficio, planteado también por la política de APS, quizá es el menos llevado a la práctica.

En 1978 se impulsa otro programa por parte de la OPS/ OMS, es el American Program Evaluation Sistems (AMPES). Este trata de canalizar todos los programas que los países tienen en el área para la consecusión de las metas que se han trazado. Así es como la relación entre las Escuelas, por medio de ASEDEE y la OPS sufrirá una modificación. A partir del AMPES ninguna relación será directa entre las instituciones educativas y la OPS. Todo deberá ser canalizado con el visto bueno de este programa, que en muchas ocasiones no dará fácil acceso a los proyectos educativos por priorizar otros aspectos considerados como los más relevantes para el logro de las metas propuestas.

\section{La Educación y la práctica de enfermería frente a la nueva tendencia de Salud}

En los últimos años en la educación de enfermería se observa un cambio con respecto a los años precedentes. A partir de 1978 se modifica la relación con la OPS/OMS, un distanciamiento se hace presente.

Además del AMPES, hay otro elemento más a mencionarse y es que la nueva asesora de enfermería de la OPS no es aceptada por las Escuelas y Facultades de enfermería como ocurrió en épocas pasadas: ellas aducen no haberla solicitado. La causa indirecta de este impase posiblemente tiene relación con el cambio de objetivos sucitado a nivel de la OPS/OMS, que en Ecuador llega más tarde que a otros países. A decir de Almeida Souza la OPS/OMS en los años 60 habla comenzado a cambiar su eje de acción en enfermería, de la educación a la prestación de serviclos. Esto es lo que sucede con la nueva asesora, que llega para la División de Enfermería del MSP y no para educación.

Se crean nuevas escuelas: Loja, Portoviejo, Machale y en Manta se abre una extensión. Esto es consecuencia de la Cadernos de Saúde Pública, R.J., 2(4): 167-196, abr/jun, 1988. 
política de Extensión de Cobertura e incremento de personal. En este caso se logra abrir unidades universitarias para capacitación del recurso en contraposición a lo acontecido en los inícios de los 70 cuando la proliferación de cursos de auxiliares de enfermería se prioriza a la creación de escuelas.

Se formula una Segunda Política de Educación, para lo cual se desarrolla un taller de discusión de la Primera. En él, la educación de enfermería comienza a ser cuestionada y los cambios que las Escuelas y Facultades inician en sus curriculae no quieren ser tan solo reajustes, se busca una transformación de fondo en la que fundamentalmente la nueva profesional tenga una formación con visión comunitaria y no exclusivamente hospitalaria, y conciba a su que hacer profesional en una práctica social, comprometida con una realidad social.

La Segunda Política de Educación, sin temor a equivocarnos, no está explicitamente incerta dentro de APS, sino dentro de la tendencia no solo de la salud comunitaria, sino también de la tendencia que está presente en la sociedad: atención a las zonas más desprotegidas de la población, fortalecimiento de un sentido crítico a partir de las necesi. dades de la población más pobre.

Pero esto en definitiva no es una contradicción ni para APS ni para la política de Extensión de Cobertura, ni para el discurso desarrollista del Estado.

Es importante anotar que las Facultades de Ciencias Médicas de las Universidades estatales se oponen a la implemen. tación de APS, por las siguientes razones:

1. APS viene a ser una prestación de servicios de mala calidad para una población que siempre ha sido marginada.

2. El MSP va a asumir la formación de recursos: auxiliares de enfermería y promotores de salud, siendo ésto responsabilidad de las universidades.

3. Resta mercado de trabajo a los profesionales que la universidad forma.

4. Se promueve el empirismo.

5. En el país no hay sistema regionalizado de servicios, aún se pone en duda la existencia de uno real sistema de salud.

Las Escuelas de Enfermería, a través de ASEDEE tampoco aceptan la APS, básicamente por las mismas causas, a excep- 
ción de la Facultad de Enfermería. Dice en un documento:

Expresión del trabajo unilateral del MSP, basado en modelos internacionales y experimentados en situaciones de salud y realidades socio-económicas, politicas distintas a las de la sociedad ecuatoriana. Representa una estrategia encaminada a realizar prestaciones oficiales de salud dirigidas a grupos poblacionales rurales y urbano marginales a través de actividades simplificadas y de bajo costo con la intervención de promotores de salud (ASEDEE; 1980; 1).

El gremio de enfermería, la Asociación Ecuatoriana de Enfermeras no se pronuncía y las enferemeras de servicio desconocen en su gran mayoría lo que es la APS.

La OPS, por suparte está permanentemente insistiendo en el papel que enfermeria juega en APS, desde el momento en que surge la Conferencia de Alma Ata. Así: la resolución XIX de la OMS del Consejo Directivo de la OPS aprobada en la $13^{\text {a }}$. Sesión Plenaria celebrada el 1 ? de octubre de 1980 sobre "Metas en Materia de Enfermería para el decenio 80/90" considera que "los programas de Extensión de Cobertura y la aplicación del concepto de APS, como estrategia para alcanzar la meta de salud para todos en el año dos mil, demanda cambios en la estructuración, preparación y utilización del personal de salud; reconocimiento que el personal de enfermería constituye el mayor potencial de recursos humanos para la provisión de atención primaria..."

Recomienda, por tanto, entre otras cosas ampliar el rol de la enfermera y definir sus funciones, incrementar al número de enfermeras y auxiliares creando nuevos puestos, que se reformulen los planes de estudio con énfasis en epidemiología, ciencias sociales, salud comunitaria, atención primaria de salud, evaluación e investigación" (OMS: 1980: 28-29).

En la 36 ava. Asemblea Mundial de la Salud, enfermería es nuevamente rescatada para APS como personal clave, no solo por su función sino como elemento que influye en la movilización de la opinión pública en favor del desarrollo eficaz de la Atención Primaria de Salud (OMS: 1983; 12).

Esto significa que enfermería puede ser potencialmente una pieza clave dentro del juego de estrategias para la salud y que el crecimiento de la profesión tiene una puerta abierCadernos de 'Saúde Pública, R.J., 2(4): 167-196, abr/jun, 1988. 
ta, que dentro de un trabajo multidisciplinario tenga poder de decisión, un status, un carácter científico, un reconocimiento económico, que ha estado buscando desde que nació.

El MSP en Ecuador, aún no integra el papel de enfermería en la nueva política en la que está envuelto. En la prestación de servicios en estos 6 años, enfermería continúa el mismo proceso de modernización y tecnologización que se opera en el resto de la sociedad. La división del trabajo en las unidades de salud, en general se acentúa más, la que se refleja en la especialización. Así en Quito y Guayaquil, los dos polos de desarrollo del país, en los hospitales más grandes se abren servicios y se reciben médicos especializados. La facultad de Ciencias Médicas de Quito, ofrece cursos de post-grado en las áreas de pediatría, ginecología, psiquiatría, salud pública, administración e investigación. Sin embargo, enfermería continúa en los hospitales afrontando el efecto de la especialización, sin que se le de opor. tunidades de perfeccionamiento académico.

Así, mientras la prestación de servicios prioriza la atención curativa, la política de APS trata de ser presentada y llevada a cabo bajo la atención preventiva con promotores de salud, en cuyo programa la enfermera realiza la formación y en mínima escala la supervisión de dicho recurso, y no cumple con acciones integrales de prestación de servicios.

De esta manera, el nuevo rol al que los organismos interna. cionales le quieren dar a enfermeria, aún no tiene cabida en la disposición de servicios del MSP. A corto plazo se verá enfrentada a un problema: el recurso se está formando dentro de una connotación comunitária, así lo atestiguan los tres curriculae de las escuelas y facultad de enfermería, sobre todo el de la ENE que está integrando los lineamientos de la APS. A este punto cabe un interrogante y es: qué práctica es la que las egresadas desarrollarán en los próximos 2 años cuando comiencen a salir las primeras promociones de enfermeras, si el MSP no transforma la prestación de servicios, haciéndolos más idóneos para incidir en los problemas de salud de la población?

Todo el proceso descrito y analizado, evidencía una preocupación en la salud comunitaria por parte del Estado, la Universitad y las Agencias Internacionales. Las dificul. tades para encontrar la solución definitiva de salud de las masas radica en la estructura misma de la sociedad, en la ideologia del Estado ecuatoriano, en el proceso histórico de conformación del Ecuador, en la crisis del capitalismo. 
In this paper, the authors describe changes in teaching and nursing practices in Ecuador, from its first step, with the introduction of foreign personnel through international agencies up to their substitution for ecuatorian professionals. At the same time, the authors study the integration of Nursing School in the structure of the University and analyse, also, hits and mistakes from the State, universities and international health organizations in the elaboration of unified sanitary policies which meet the needs of the population. Finally, the role of nursing altogether is discussed.

\section{REFERÊNCIAS BIBLIOGRÃFICAS}

AGUIRRE, Manuel Augustín, La Segunda Reforma Universitaria. Quito, Ed. Universitaria, 1973.

Agencia para el Desarrollo Internacional (AID). Alianza para el Progreso; 30 años de colaboración técnica y económica, entre Ecuador y E.U.: 1942.1972. Quito, Departamento de Pu blicaciones Fecose, 1972.

Alianza para el Progreso. Carta de Punta del Este. 1961.

ANDRADE, Jorge, "Marco Conceptual de la Educación Médica en América Latina", en Educación Médica y Salud. OPS, Vol. 12 No: 2, Washington, OPS, 1978.

ASEDEE, Necesidades Cuantitativas presentes y futuras del personal de enfermeria para los servicios de Salud del Ecuador, Primer Seminario de Educación de Enfermeria Cuenca, Escuela de Enfermaria de la Universidad de Cuenca, 1969.

ASEDEE, Oferta y Demanda del Personal de Enfermeriadel Ecuador, Segundo Seminario Nacional de Educación de Enfermeria, Guayaquil, Escuela de Enfermeria de la Universidad Estatal de Guayaquil, 1971.

ASEDEE, Aspectos de la problemática de la Educación de Enfermeria en el pais y sus posibles soluciones, Quito, (mimeo), 1971.

ASEDEE, Formación de Auxiliares de Enfermeria del Ecuador. Tercer Seminario Nacional de Educación de Enfermería, Quito, Escuela Nacional de Enfermería de la Universidad Central del Ecuador, 1972.

ASEDEE, Políticas de Educación de Enfermeria en el Ecuador y Normas minimas para la organización y desarrollo de organismos universitarios de Educación de Enfermeria, Guayaquil, Mimeo, 1972.

ASEDEE, Integración docencia servicio para la formación de la Enfermera que el Ecuador necesita, Cu arto Seminario Nacional de Educación de Enfermería, Cuenca, Escuela de Enfermeria de la Universidad de Cuenca, 1973.

ASEDEE, Evaluación del Año de Salud Ru ral de las egresadas de Enfermeria en el Ecuador: promociones 1973-1974, Cuarto Seminario Nacional de Educación de Enfermería, Quito. Facultad de

Cadernos de Saúde Pública, R.J., 2(4): 167-196, abr/jun, 1988. 
Enfermeria de la Pontificia Universidad Católica del Ecuador, 1975.

ASEDEE, Politica de Educación de Enfermeras del Ecuador, Quito, (mimeo) 1979.

ASEDEE, Taller Seminario de Evaluación de la politica Educativa, Quito, 1979.

ASEDEE, Posición de la Asociación Ecuatoriana de Escuela de Enfermeria frente al Proyecto de Educación Integral de Cobertura: Servicios de Atención familiar e investigación del Ministerio de Salud Pública, Quito, 1980.

ASEDEE, MSP, IESS, "Oferta y Demanda del Personal de Enfermería en la Década $1972-1981$ y las proyecciones hasta $1985^{\prime \prime} . P o$. nencia al II Congreso Nacional de Enfermeras, Guayaquil, 1982.

ASEDEE, Comunicaciones recibidas y enviadas 1969 a 1982, Qu ito, Archivos ASEDEE, 1983.

ASEDEE, Informes de las Presidentas, 1969 a 1972. Quito, Archivos ASEDEE, 1983.

ASEDEE, Cronogramas de Trabajo del Directorio de los años 1973 a 1979, Quito, Archivos de ASEDEE, 1983.

ASEDEE, Libro de Actas de reunionesde Directorio de los Años 1979 a 1982. Quito, Archivos ASEDEE, 1983.

ASEDEE, Libro de Actas de las Kuntas Directivas y de Asambleas de los años 1979 a 1982, Quito, Archivos ASEDEE, 1983.

BARSKY, Osvaldo, et al. Pollticas Agrarias, Colonización y Desarro. llo Ru ral en el Ecuador, Qu ito, OEA/CEPLAES, 1982.

BOCCO, A. Fernández, I., Moncayo P., et al. Ecuador: el Mito del Desarrollo, Qu ito, Ed. El Conejo/ILDIS, 1982.

BUNGE, MÁRIO, La Investigación Cienttfíca, 2 ed., Barcelona, Ed. Ariel, 1983.

CARRILLO, GEORGINA, La Integración Docente-Asistencial: Movimientos en Salud que la precedieron, La problemática actual de Enfermeria, Quito, mimeo, 1982.

CASTILLO, GLORYA y Monge, Dellia, Análisis de la Práctica de la Enfermera Egresada de las Escuelas Estatal y Católica de Guayaquil dentro del Plan Nacional de Salud Rural teniendo como base la formación universitaria, Tesis de Grado, Quito, Facultad de Enfermería PUCE, 1983.

Consejo Nacional de Desarrollo (CONADE), Plan Nacional de Desarrollo 1980-1984 del Gobierno Democrático, II Parte, Tomo III Pollticas y Programas Sectoriales: desarrollo social, empleo, salarios, capacitación de recursos humanos promoción popular y vivienda, Otavalo, Ed. Gallo Capitán, sn.f.

CUEVA, AGUSTIN, El Proceso de Dominación Politica en el Ecuador, Quito, Ed. Alberto Crespo, 1981.

DE ALMEIDA SOUZA, Alina María, Desenvolvimiento dos Serviços de Asesoria de Enfermagen Latino-Americana (1940-1980), Salvador, Bahia, Brasil, Bu seau Gráfica e Editora Letata, 1982.

DE LA TORRE, Patrícia, La Participación Gremial de Enfermeria: La Asociación Ecuatoriana de Enfermeras 19581982, Quito, mimeo, 1982.

DE LA TORRE, Patrícia, El Estado y Salud; situación y alternativas: el caso Columbe, Chimborazo, Quito, mimeo, 1982.

Escuala de Enfermería de La Universidad de Guayaquil, Evaluación de los Programas Educativos de Enfermeria, Guayaquil (mimeo), 1970.

Escuela San Vicente de Paúl, Algunas consideraciones para la refor. mulación de la Politica educativa de Enfermeria en el Ecuador, Guayaquil, Universidad Católica de Santiago de Guayaquil, 1979. 
Escuela de Enfermería de Guayaquil, "Análisis de la Poltica de Educación de Enfermeria", Guayaquil, Universidad de Guayaquil, 1979.

Escuela Nacional de Enfermería, Análisis y recomendaciones de la Politica educativa de enfermeria y normas minimas para la orga. nización y desarrollo de organismos universitarios de educación en Enfermeria, Quito, Universidad Central del Ecuador, 1979.

Escuela de Enfermerta, Evaluación y replanteamiento de la Politica de Educación de Enfermería del Ecuador, Cuenca, Facultad de Ciencias Médicas, Universidad de Cuenca, 1979.

Escuela Nacional de Enfermería, Plan Curricular, Quito, Facultad de Ciencias Médicas, Universidal Central, 1981.

Facultad de Enfermeria-PUCE, Historia de la Facultad de Enfermería, Quito, Archivos, Facultad Enfermería, 1969.

Facultad de Enfermería, Evaluación de Polftica de Educación de Enfermeria y las normas minimas para la organización y desarrollo de organismos universitarios de Educación de Enfermeria, Quito, PUCE, 1979.

MAHLER, Haljdan, El sentido de "la salud para todos en el año 2000' en Foro Mundial de la Salud, 2 (1), Ginebra, OMS, 1981.

MALO, Hemán, História del Renacimiento Universitario Ecuatoriano, Vol. I, Quito, Corporación Editora Nacional, Sn $\mathrm{f}$.

Ministerio de Salud Pública del Ecuador, Extensión de Cobertura de los Servicios de Salud, Ponencia del Ecuador en IV Reunión Especial de Ministros de Salud de las Américas, Washington, D.C. 26-27 de Septiembre, Quito, Editora Mantilla, 1977.

Ministerio de Salud Pública, MSP, Informe de Labores (1972-1977, Quito, 1977.

MSP/FODERUMA, Atención Primaria de Salud con participación comunitaria. Programa de Extensión de Cobertura, Manual de Uso Institucional, Vol. I, Quito, Ecuador, Gráficas San Pablo Cia. Ltda. 1979.

MSP, "Política, Propósitos y Objetivos de la Atención Primaria de Salud, funciones y tareas del Promotor de Salud" en Informe Fi. nal de Seminario de Revisión de los Manuales de Atención Pri. maria de Salud, Qu ito, MSP mimeo, 1981.

MONT aLVO, Martha y Suárez, Alkinza, A nálisis de la Práctica de la egresada de la Escuela Nacional de Enfermerta de Quito, dentro del Plan Nacional de Salud Ru ral, Qu ito, Facultad de Enfermería PUCE, Tesis de Grado, 1983.

MOREANO, A Moncada, José et al., Ecuador: Pasado y Presente, Quito, Universidad Central del Ecuador, Institu to de Investigaciones Económicas, 1976.

MORILlO, Rocío y Ríos, Nelly, Análisis de la Práctica de la Enfermera egresada de la Escuela Estatal de Cuenca dentro del Plan Nacional de Salud Rural, Quito, Facultad de Enfermería, PUCE, Tesis de Grado, 1983.

OMS/OPS, Metas en materia de enfermería, Ginebra, Resolución XIX, 13a. Sesión Plenaria, 1980.

OMS/OPS, "Función del Personal de Enfermería y de las partes en la estretegia de salud para todos", Resolución de la Asamblea Mundial de la Salud, Ginebra, 36 Asamblea Mundial de la Salud, 12a. Sesión Plenaria, 1983.

OPS/OMS, Comité de Expertos de la OPS/OMS en la enseñanza de Enfermerla Médico Quirírgica en las Escuelas de Enfermeria de América Latina, Primer Informe, Publicación Cien tífica No. 242, Washington, OPS, 1972.

OPS/OMS, Plan Decenal de Salud para las Américas, Documento Oficial No. 118, Washington, OPS/OMS, 1973.

Cadernos de Saíde Pública, R.J., 2(4): 167-196, abr/jun, 1988. 
OPS/OMS, Seminario sobre Enseñanza de Enfermeria a nivel universitario, Informe Final, Lima, Peru, 22 de noviembre. 3 de diciembre de 1971, Publicación Científica No. 259, Washington, OPS, 1973.

OPS/OMS, Enseñanza de Enfermerta integrada a la educación media: consideraciones generales sobre planificación y desarrollo de programas, Pu blicacípon Científica No. 274, Washington, 1973.

OPS/OMS, Informe del Comité del Programa de Textos de la OPS/ OMS para la enseñanza de Enfermerla en Salud Comunitaria, Washington D.C. 21 de julio - 8 de agosto de 1975, Publicación Científica No. 332, Washington, 1976.

OPS/OMS, Informe del Comité del Programa de Libros de Tex to de la OPS/OMS para la enseñanza de la Introducción a la Enfermerta, Washington, D.C., 5 al 9 de noviembre de 1973, Publicación Científica No. 303, Washington, 1975.

OPS/OMS, Boletin Informativo, Enero, 1977.

OPS/OMS, Atención Primaria de Salud, Informe de la Conferencia Internacional sobre Atención Primaria de Salud, Alma-Alta, URSS, sep 1978, Ginebra, OMS, 1978.

OPS/OMS, Informe del Comité del Programa de Tex tos de la OPS/ OMS para la enseñanza de enfermería en Salud Mental y Psiquia. tria, Washington, D.C. 23 de septiembre -1 de octubre de 1976, Publicación No. 363, Washington, 1978.

RIFFKA, Sohel, comp, Los modelos de la crisis, Quito, Ed. El Conejo/ILDIS, 1983.

RODRIGUEZ NIETO, Eleuterio, Integracao Docente-Assistencial en Saide, Disetocao de Maestrado Apresentoda a Facultade de Medicina da Universidade de São Paulo Departamento de Medicina Preventiva, São Paulo, mimeo, 1979.

ROJAS, Piedad H. de la C., Papel de la Asociación Ecuatoriana de Escuelas de Enfermeria: la Politica de Educación de Enfermeria del Ecuador. Qu inquenio 1979-1984, Qu ito, Asociación Ecuatoriana de Escuelas de Enfermería, 1983.

SUÁREZ, José y López, Raúl, Evaluación del Plan Nacional de Sa. lud Rural, Institu to de Investigaciones Nu tricionales Médico-Sociales del MSP (ININMS), Quito, 1982.

VELASCO, Fernando, Ecuador: subdesarrollo y dependencia, QuF to, Ed. El Conejo, 1981.

VELASCO, Margarita, Enfermeria: Luchas y Organización Gremial, Qu ito, 1981.

VELASCO MARGARITA, et al, Determinantes históricos de la Práctica de Enfermeria en el Hospital Eugenio Espejo, Quito, Hospital Lugenio Espejo, 1982.

VERDERESE, Olga y Plant, Renata, Informe del Estudio de las Escuelas Universitarias de Enfermeria en América Latina, Informe de Enfermería, Washington, OPS, No. 18, 1976. 